\title{
Pure injective and $*$-pure injective LCA groups
}

\author{
Peter Loth
}

ABstRaCT - A proper short exact sequence $0 \rightarrow A \rightarrow B \rightarrow C \rightarrow 0$ in the category $\mathbb{\Omega}$ of locally compact abelian (LCA) groups is called $*$-pure if the induced sequence $0 \rightarrow A[n] \rightarrow B[n] \rightarrow C[n] \rightarrow 0$ is proper exact for all positive integers $n$. An LCA group is called $*$-pure injective in $\mathfrak{Q}$ if it has the injective property relative to all *-pure sequences in $\mathcal{R}$. In this paper, we give a complete description of the $*$-pure injectives in $\mathbb{R}$. They coincide with the injectives in $\mathbb{R}$ and therefore with the pure injectives in $\mathfrak{R}$. Dually, we determine the topologically pure projectives in $\mathfrak{R}$.

Mathematics Subject Classification (2010). 20K35, 22B05, 20K25, 20K40, $20 \mathrm{~K} 45$.

KEYworDs. Locally compact abelian groups, pure injectives, *-pure injectives, topologically pure projectives.

\section{Introduction}

All groups considered in this paper are Hausdorff abelian topological groups and they will be written additively. For a group $G$ and a positive integer $n$, let $n G=\{n x: x \in G\}$ and $G[n]=\{x \in G: n x=0\}$. Let $\mathfrak{R}$ denote the category of locally compact abelian groups with continuous homomorphisms as morphisms. In [15], Moskowitz developed a homological theory in the category $\mathfrak{Z}$ and studied the functors Hom, $\otimes$, Tor and Ext on certain subcategories of $\mathbb{2}$. Later Fulp and Griffith ([9], [10]) extended Moskowitz's construction of the functor Ext to the category $\mathfrak{\Omega}$. Following Fulp and Griffith ([9]), we call a morphism proper if it is open onto its image. An exact sequence

$$
G_{1} \stackrel{\phi_{1}}{\longrightarrow} G_{2} \stackrel{\phi_{2}}{\longrightarrow} \ldots \stackrel{\phi_{n}}{\longrightarrow} G_{n}
$$

(*) Indirizzo dell'A.: Department of Mathematics, Sacred Heart University, 5151 Park Avenue, Fairfield, Connecticut 06825, USA.

E-mail: lothp@sacredheart.edu 
in $\mathfrak{Z}$ is called proper exact if each morphism $\phi_{i}$ is proper. A proper short exact sequence $E: 0 \rightarrow A \rightarrow B \rightarrow C \rightarrow 0$ in $\mathfrak{Q}$ is called an extension of $A$ by $C$ (in $\mathfrak{R}$ ) and $\operatorname{Ext}(C, A)$ denotes the group of extensions of $A$ by $C$ (see [9]). Then the extension $E$ is pure if and only if the induced sequence

$$
E_{n}: 0 \rightarrow A[n] \rightarrow B[n] \rightarrow C[n] \rightarrow 0
$$

is exact for all positive integers $n$ (see [6, Theorem 29.1]). The elements represented by pure extensions of $A$ by $C$ form a subgroup of $\operatorname{Ext}(C, A)$ which is denoted by $\operatorname{Pext}(C, A)$. If each sequence $E_{n}$ is proper exact, we call the extension $E *$-pure.

The concept of purity plays an important role in abelian group theory (see for instance [6]). In [7], Fulp studied pure extensions in the category 2 . As it was pointed out by Armacost [1], much of the paper is based on [7, Proposition 2] (stating that the dual of a pure extension is pure) which is unfortunately not valid for all groups in $\mathrm{R}$.

In this paper, we continue our study of $*$-pure extensions started in [13] and give a complete description of the $*$-pure injectives in the category of locally compact abelian groups. Let $\widetilde{C}$ denote the class of all groups $X$ in $\mathfrak{Z}$ such that $X$ is connected or $X$ is a torsion-free group which is either discrete or a topological torsion group (for the definition, see Section 2). Then a group $G$ in $\mathfrak{Q}$ has the property that every *-pure extension of $G$ by a group in $ᄃ$ splits if and only if $G$ has the form $R \oplus T$ where $R$ is a vector group and $T$ is a toral group (Theorem 3.7). Consequently, the $*$-pure injectives in $\mathbb{2}$ coincide not only with the injectives in $\mathbb{2}$ but also with the pure injectives in $\mathbb{Q}$ (see Theorem 4.1 and Corollary 4.2). Recall that a proper exact sequence $0 \rightarrow A \rightarrow B \rightarrow C \rightarrow 0$ in $\mathfrak{Q}$ is said to be topologically pure if for each positive integer $n$, the induced sequence

$$
0 \rightarrow \overline{n A} \rightarrow \overline{n B} \rightarrow \overline{n C} \rightarrow 0
$$

is proper exact (see [13]). Using Pontrjagin duality, we obtain the following result: A group in 2 is topologically pure projective if and only if it has the form $R \oplus F$ where $R$ is a vector group and $F$ is a free group (see Corollary 4.3).

The group of real numbers with the usual topology is denoted by $\mathbb{R}, Z$ is the group of integers, $Q$ is the group of rationals taken discrete and $T$ denotes the quotient $\mathbb{R} / Z$. By Z $\left(p^{\infty}\right)$ we mean the quasicyclic group and $F_{p}$ is the additive group of the $p$-adic number field with the usual topology. For any groups $G$ and $H$ in $\mathcal{R}$, let $\operatorname{Hom}(G, H)$ denote the group of all continuous homomorphisms from $G$ to $H$. The identity component of $G$ is given by $G_{0}$ and the union of all compact subgroups of $G$ is denoted by $B(G)$. Notice that $B(G)$ is a closed subgroup of $G$ (cf. [4, Proposition 3.3.6]). 
The Pontrjagin dual of $G$ is

$$
\widehat{G}=\operatorname{Hom}\left(G,{ }^{\prime T}\right),
$$

endowed with the compact-open topology. All isomorphisms are understood to be topological isomorphisms and all considered direct sums are topological direct sums. We mostly follow the standard notation in [6] for abelian groups and [1] for locally compact abelian groups. For background information on abelian topological groups and Pontrjagin duality, we refer the reader to the books [4] and [12].

\section{Preliminaries}

A group $G$ in $\mathbb{R}$ is called injective in $\mathbb{R}$ if for every proper exact sequence $0 \rightarrow A \rightarrow B \rightarrow C \rightarrow 0$ in $\mathfrak{R}$ and every $\alpha \in \operatorname{Hom}(A, G)$ there is a $\beta \in \operatorname{Hom}(B, G)$ such that the diagram

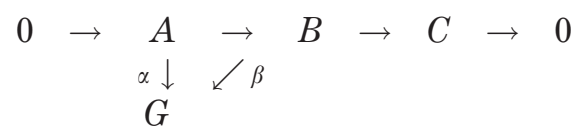

is commutative. Dually, $G$ is called projective in $\mathbb{Q}$ if for every proper exact sequence $0 \rightarrow A \rightarrow B \rightarrow C \rightarrow 0$ in $\mathfrak{Q}$ and every $\gamma \in \operatorname{Hom}(G, C)$ there is a $\delta \in \operatorname{Hom}(G, B)$ such that the diagram

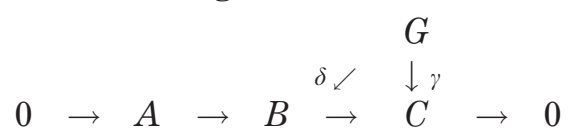

is commutative. Dixmier [3] and later Moskowitz [15] independently characterized the injectives in $\mathbb{R}$ :

THEOREM 2.1 ([3],[15]). The following are equivalent for a group $G$ in $\mathbb{2}:$

(1) $G$ is injective in $\mathrm{R}$;

(2) $G \cong \mathbb{R}^{n} \oplus^{m} \mathbb{T}^{\mathrm{m}}$ where $n$ is a nonnegative integer and $\mathrm{m}$ is a cardinal.

Using Pontrjagin duality, Moskowitz [15] proved the following:

THEOREM 2.2 ([15]). The following are equivalent for a group $G$ in $\mathbb{R}$ :

(1) $G$ is projective in $\mathrm{Q}$;

(2) $G \cong \mathbb{R}^{n} \oplus \bigoplus_{\mathfrak{m}}$ Z where $n$ is a nonnegative integer and $\mathfrak{m}$ is a cardinal. 
Using the notion of proper morphisms, Fulp and Griffith [9] developed the (discrete) group-valued extension functor Ext for the category $\mathfrak{Z}$, generalizing both the functor Ext as defined in (discrete) abelian group theory and the functor Ext studied by Moskowitz [15]. We would like to point out the unfortunate fact that, at the same time, another use of the term "proper" exists; it is used by some authors in topology as a synonym for stably closed (what Engelking [5] calls "perfect"). The following basic properties will be useful:

Proposition 2.3 ([9]). If $G$ is a discrete group, then $\operatorname{Ext}(\top, G) \cong G$. Hence the range of Ext is all of the discrete groups.

Theorem 2.4 ([9]). Let $G$ be a group in 2 . If $\left\{H_{i}: i \in I\right\}$ is a collection of groups in $\mathbb{2}$ such that $H_{i}$ is compact for almost all $i$, then $\operatorname{Ext}\left(G, \prod_{i \in I} H_{i}\right) \cong \prod_{i \in I} \operatorname{Ext}\left(G, H_{i}\right)$.

In [9], Fulp and Griffith proved that the Hom-Ext sequences are exact except possibly at the right end. Then, in [10], they showed that Ext is right-exact; in fact, it was shown that $\mathrm{Ext}^{n}=0$ for all $n \geq 2$.

Theorem 2.5 ([9],[10]). Let $G$ be a group in $\mathfrak{Q}$ and let $0 \rightarrow A \rightarrow$ $B \rightarrow C \rightarrow 0$ be a proper exact sequence in $\mathbb{R}$. Then the following induced sequences are exact:

(1) $0 \rightarrow \operatorname{Hom}(G, A) \rightarrow \operatorname{Hom}(G, B) \rightarrow \operatorname{Hom}(G, C) \rightarrow \operatorname{Ext}(G, A) \rightarrow$

$$
\operatorname{Ext}(G, B) \rightarrow \operatorname{Ext}(G, C) \rightarrow 0,
$$

(2) $0 \rightarrow \operatorname{Hom}(C, G) \rightarrow \operatorname{Hom}(B, G) \rightarrow \operatorname{Hom}(A, G) \rightarrow \operatorname{Ext}(C, G) \rightarrow$

$$
\operatorname{Ext}(B, G) \rightarrow \operatorname{Ext}(A, G) \rightarrow 0 .
$$

Using the right-exactness of Ext, Fulp and Griffith were able to improve Theorem 2.1:

THeOREm 2.6 ([10]). The following are equivalent for a group $G$ in $\mathrm{R}:$

(1) $G \cong \mathbb{R}^{n} \oplus T^{\mathfrak{m}}$ where $n$ is a nonnegative integer and $\mathfrak{m}$ is a cardinal.

(2) $\operatorname{Ext}(C, G)=0$ for all connected groups $C$ in $\mathbf{R}$.

A group $G$ in $\mathcal{Q}$ is called a topological torsion group if $\lim _{n \rightarrow \infty} n ! x=0$ for all $x \in G$. Robertson [16] established several characterizations of topological torsion groups including the following: 
THEOREM 2.7 ([16]). A group $G$ in $\mathfrak{\Omega}$ is a topological torsion group if and only if both $G$ and $\widehat{G}$ are totally disconnected.

Now let $\left\{G_{i}: i \in I\right\}$ be a collection of groups in $\mathfrak{R}$ and let $H_{i}$ be a compact open subgroup of $G_{i}$ for every $i \in I$. Then the local direct product of the groups $G_{i}$ with respect to the subgroups $H_{i}$ is defined to be the group

$$
G=\left\{\left(x_{i}\right) \in \prod_{i \in I} G_{i}: x_{i} \in H_{i} \text { for almost all } i\right\}
$$

and is topologized so that it contains $\prod_{i \in I} H_{i}$ (with its compact product topology) as an open subgroup (cf. [12, (6.16)]). The group $G$ is in $\mathfrak{R}$ and is denoted by $L P_{i \in I}\left(G_{i}, H_{i}\right)$. Braconnier [2] and Vilenkin [18] proved independently that every topological torsion group $G$ can be decomposed into a local direct product of its $p$-components

$$
G_{p}=\left\{x \in G: \lim _{n \rightarrow \infty} p^{n} x=0\right\}
$$

belonging to different primes $p$ :

Theorem 2.8 ([2], [18]). Let $G$ be a topological torsion group and let $H$ be any compact open subgroup of $G$. Then $G_{p}$ is a closed subgroup of $G$ for every prime $p$ and $G$ is isomorphic to the local direct product $L P_{p \in \mathbf{P}}\left(G_{p}, H_{p}\right)$.

Let $p$ be a prime and $G$ a group in $\mathrm{I}$. Then $G$ is called a topological $p$ group if $G=G_{p}$. If $G$ contains a dense divisible subgroup, then $G$ is said to be densely divisible (see [16]). The next result will be needed:

Proposition 2.9 ([1]). Let $G$ be a nontrivial topological p-group. If $G$ is densely divisible, then $G$ contains a closed subgroup $D$ such that $D \cong F_{p}$ or $D \cong Z\left(p^{\infty}\right)$.

\section{Splitting $*$-pure extensions}

For groups $A$ and $C$ in $\mathcal{R}$, let ${ }^{*} \operatorname{Pext}(C, A)$ denote the set of elements $E \in \operatorname{Ext}(C, A)$ such that $E$ is equivalent to some $*$-pure extension of $A$ by $C$. Then ${ }^{*} \operatorname{Pext}(C, A) \subseteq \operatorname{Pext}(C, A)$ and ${ }^{*} \operatorname{Pext}(C, A)=0$ if and only if every *-pure extension of $A$ by $C$ splits (cf. [13]). 
LEMMA 3.1. Let $A$ and $C$ be groups in $\mathbb{2}$. Then:

(1) If $C$ is torsion-free, then ${ }^{*} \operatorname{Pext}(C, A)=\operatorname{Ext}(C, A)$.

(2) If $A=H \oplus K$ for some groups $H$ and $K$ in $\Omega$ and ${ }^{*} \operatorname{Pext}(C, A)=0$, then ${ }^{*} \operatorname{Pext}(C, H)=0$.

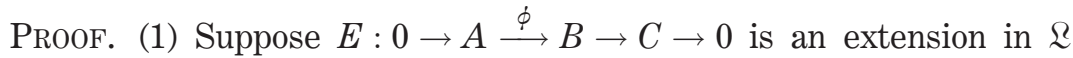
where $C$ is torsion-free. Then $E$ is pure and $C[n]=0$ for every positive integer $n$. Since $\phi$ is proper and injective, each map $\left.\phi\right|_{A[n]}: A[n] \rightarrow B[n]$ is proper. It follows that each sequence $0 \rightarrow A[n] \rightarrow B[n] \rightarrow C[n] \rightarrow 0$ is proper exact, hence $E$ is $*$-pure.

(2) Let $E: 0 \rightarrow H \stackrel{\psi}{\longrightarrow} B \rightarrow C \rightarrow 0$ be a $*$-pure sequence. Then

$$
E^{\prime}: 0 \rightarrow H \oplus K \rightarrow B \oplus K \rightarrow C \rightarrow 0
$$

is an extension in $\mathfrak{Q}$ and $0 \rightarrow H[n] \oplus K[n] \rightarrow B[n] \oplus K[n] \rightarrow C[n] \rightarrow 0$ is proper exact for all positive integers $n$. If the sequence $E^{\prime}$ splits, then $\psi(H) \oplus K$ is a direct summand of $B \oplus K$. But then $\psi(H)$ is a direct summand of $B$ (see the proof of [1, Lemma 9.11]), hence the sequence $E$ splits.

The proof of [13, Theorem 4.3(1)] shows the following:

Proposition 3.2. If a group $G$ in 2 satisfies ${ }^{*} \operatorname{Pext}(C, G)=0$ for all connected groups $C$ in $\mathfrak{R}$, then there is a closed subgroup $H$ of $G$ such that $G=G_{0} \oplus H$ and $G_{0} \cong \mathbb{R}^{n} \times T^{\mathfrak{m}}$ for some nonnegative integer $n$ and cardinal $\mathfrak{m}$.

Let $E: 0 \rightarrow A \stackrel{\phi}{\longrightarrow} B \rightarrow C \rightarrow 0$ be a proper exact sequence in $\mathbb{R}$ and $\alpha \in \operatorname{Hom}(A, G)$ where $G$ is a group in $\mathbb{2}$. Then there is a standard pushout diagram for $\alpha$ and $\phi$

$$
\begin{array}{cccccccccc}
E: \quad 0 & \rightarrow & A & \stackrel{\phi}{\longrightarrow} & B & \stackrel{\pi}{\longrightarrow} & C & \rightarrow & 0 \\
& & & \downarrow & & \downarrow & & \| & & \\
\alpha E: \quad 0 & \rightarrow & G & \stackrel{\phi^{\prime}}{\longrightarrow} & X & \stackrel{\pi^{\prime}}{\longrightarrow} & C & \rightarrow & 0
\end{array}
$$

(cf. [9, Proposition 2.5]). Recall that $X=(G \oplus B) / N$ where $N=$ $\{(-\alpha(a), \phi(a)): a \in A\}$ is a closed subgroup of $G \oplus B, \phi^{\prime}: g \mapsto(g, 0)+N$ and $\pi^{\prime}:(g, b)+N \mapsto \pi(b)$. Further, $\alpha E$ is a proper exact sequence in $\mathbb{Z}$ (see [9, p. 350]). The next result will be useful: 
LEMMA 3.3. Let $E: 0 \rightarrow A \stackrel{\phi}{\longrightarrow} B \stackrel{\pi}{\longrightarrow} C \rightarrow 0$ be a proper exact sequence in $\mathbb{\Omega}$ such that $A$ is divisible and $B[n]$ is $\sigma$-compact for all positive integers $n$. Suppose that $G$ is a group in $\mathfrak{R}$ and $\alpha \in \operatorname{Hom}(A, G)$. Then both $E$ and $\alpha E$ are *-pure.

Proof. Let $n$ be a positive integer. The exact sequence $E$ is pure because $A$ is divisible, therefore the induced sequence

$$
E_{n}: 0 \rightarrow A[n] \stackrel{\left.\phi\right|_{A[n]}}{\longrightarrow} B[n] \stackrel{\left.\pi\right|_{B[n]}}{\longrightarrow} C[n] \rightarrow 0
$$

is exact. The map $\left.\phi\right|_{A[n]}$ is proper and since $B[n]$ is $\sigma$-compact, $\left.\pi\right|_{B[n]}$ is proper by the open mapping theorem (see [12, (5.29)]), hence $E_{n}$ is proper exact. Therefore, $E$ is $*$-pure. The maps $\alpha$ and $\phi$ have a standard pushout diagram

$$
\begin{array}{rllrllllll}
E: \quad 0 & \rightarrow & A & \stackrel{\phi}{\longrightarrow} & B & \stackrel{\pi}{\longrightarrow} & C & \rightarrow & 0 \\
& & & \downarrow & & \downarrow & & \| & & \\
\alpha E: \quad 0 & \rightarrow & G & \stackrel{\phi^{\prime}}{\longrightarrow} & X & \stackrel{\pi^{\prime}}{\longrightarrow} & C & \rightarrow & 0
\end{array}
$$

and $\alpha E$ is proper exact. Since $E$ is pure, the sequence $\alpha E$ is pure ([11, Lemma $26])$, hence the induced sequence $(\alpha E)_{n}: 0 \rightarrow G[n] \rightarrow X[n] \rightarrow C[n] \rightarrow 0$ is exact. We need to show that $(\alpha E)_{n}$ is proper exact. Notice that the continuous surjective homomorphism $\varphi=\left.\pi^{\prime}\right|_{X[n]}: X[n] \rightarrow C[n]$ is open if and only if the induced map $\bar{\varphi}: X[n] / \operatorname{ker} \varphi \rightarrow C[n]$ is an isomorphism in $\mathfrak{Q}$ (cf. [12, p. 41]). The group $N=\{(-\alpha(a), \phi(a)): a \in A\}$ is divisible and therefore pure in $G \oplus B$, hence $X[n]=((G \oplus B) / N)[n]=(G[n] \oplus B[n]+N) / N$. Notice that both $G[n] \oplus B[n]+N$ and $G[n] \oplus 0+N$ are locally compact since $X[n]$ and $\operatorname{ker} \varphi=(G[n] \oplus 0+N) / N$ are locally compact $([12,(5.25)])$. The group $X[n] / \operatorname{ker} \varphi$ is equal to

$$
\frac{(G[n] \oplus B[n]+N) / N}{(G[n] \oplus 0+N) / N} \cong \frac{G[n] \oplus B[n]+N}{G[n] \oplus 0+N}=\frac{(0 \oplus B[n])+(G[n] \oplus 0+N)}{G[n] \oplus 0+N}
$$

(cf. $[12,(5.35)]$ ) and by the second isomorphism theorem in $\mathbb{2}$ (see [9, Theorem 3.3]), the latter group is isomorphic to

$$
\frac{0 \oplus B[n]}{(0 \oplus B[n]) \cap(G[n] \oplus 0+N)}=\frac{0 \oplus B[n]}{0 \oplus \phi(A[n])} \cong C[n]
$$

since $B[n]$ is $\sigma$-compact. Thus we have an isomorphism from $X[n] / \operatorname{ker} \varphi$ to $C[n]$ given by $((g, b)+N)+\operatorname{ker} \varphi \mapsto \pi(b)(g \in G[n], b \in B[n])$. Since this map coincides with $\bar{\varphi}$ it follows that $\varphi: X[n] \rightarrow C[n]$ is open. Therefore, $\alpha E$ is $*$-pure. 
PROPOSITION 3.4. Let $G$ be a totally disconnected group in $\mathbb{2}$ such that $\operatorname{Ext}(\widehat{Q}, G)=0$. Then $G$ is a topological torsion group.

Proof. By Theorem 2.7, it suffices to show that $\widehat{G}$ is totally disconnected. To prove this, we argue as in the proof of [14, Theorem 2.7 (ii) $\Rightarrow$ (iii)]. First, notice that the quotient $G / B(G)$ is discrete (cf. [12, (9.26)(a)]) and torsion-free, and that $(\mathrm{Q} / \mathrm{Z})^{-}$is compact since $\mathrm{Q} / \mathrm{Z}$ is discrete $([12$, (23.17)]). The proper exact sequence $0 \rightarrow B(G) \rightarrow G \rightarrow G / B(G) \rightarrow 0$ gives rise to the exact sequence $0=\operatorname{Ext}(\widehat{Q}, G) \rightarrow \operatorname{Ext}(\widehat{Q}, G / B(G)) \rightarrow 0$. But then exactness of the sequence

$$
0=\operatorname{Hom}((\mathrm{Q} / \mathrm{Z}) \widehat{,}, G / B(G)) \rightarrow \operatorname{Ext}(\widehat{Z}, G / B(G)) \rightarrow \operatorname{Ext}(\widehat{\mathbb{Q}}, G / B(G))=0
$$

yields $G / B(G) \cong \operatorname{Ext}(\widehat{Z}, G / B(G))=0$ by Proposition 2.3 , thus $G$ coincides with $B(G)$. Since $\widehat{G}_{0}$ is the annihilator of $B(G)$ in $\widehat{G}$ (cf. [12, (24.17)]), it follows that $\widehat{G}$ is totally disconnected.

The following lemma will be needed:

Lemma 3.5 [14, Lemma 2.6]. Suppose that $G$ is a group in 2 possessing a compact open subgroup. Then $G$ is densely divisible if and only if $G / C$ is divisible for every compact open subgroup $C$ of $G$.

Proposition 3.6. Suppose that $G$ is a topological torsion group such that $\operatorname{Ext}(X, G)=0$ for every torsion-free group $X$ in $\mathbb{2}$ which is either discrete or a topological torsion group. Then $G$ is densely divisible.

Proof. Our proof is similar to the second part of the proof of [8, Theorem 7]. Let $C$ be a compact open subgroup of $G$ and set $A=G / C$. Then for any torsion-free group $X$ in $\mathbb{Q}$ which is discrete or a topological torsion group, exactness of the sequence

$$
0=\operatorname{Ext}(X, G) \rightarrow \operatorname{Ext}(X, A) \rightarrow 0
$$

yields $\operatorname{Ext}(X, A)=0$. Recall that a discrete group $H$ is said to be cotorsion if $\operatorname{Ext}(J, H)=0$ for every discrete torsion-free group $J$ (see [6, page 232]). Then the group $A$ is cotorsion. Since $A$ is also torsion, we have $A=B \oplus D$ for some bounded group $B$ and divisible group $D$ (see [6, Corollary 54.4]). A bounded group is a direct sum of cyclic groups ([6, Theorem 17.2]), so if $B \neq 0$, then $B$ contains a direct summand $B^{\prime} \cong Z / p^{n} Z$ for some prime $p$ and positive integer $n$. By [13, Example 2.4], there is a non-splitting 
proper exact sequence

$$
0 \rightarrow B^{\prime} \rightarrow K \rightarrow L \rightarrow 0
$$

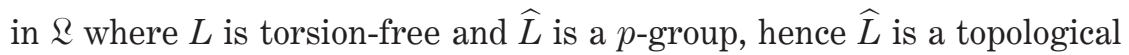
torsion group. By Theorem 2.7, $L$ is a topological torsion group and we have $\operatorname{Ext}(L, A)=0$. But then Theorem 2.4 shows that $\operatorname{Ext}\left(L, B^{\prime}\right)=0$ which is impossible. Therefore $B=0$ and it follows from Lemma 3.5 that $G$ is densely divisible.

Let 5 denote the class of groups $X$ in $\mathbb{R}$ such that $X$ is connected or $X$ is a torsion-free group which is either discrete or a topological torsion group. Then the groups $G$ in 2 having the property that every *-pure extension of $G$ by a group in 5 splits can be characterized as follows:

Theorem 3.7. A group $G$ in 2 satisfies ${ }^{*} \operatorname{Pext}(X, G)=0$ for all groups $X$ in 5 if and only if $G \cong \mathbb{R}^{n} \times \mathbb{T}^{\mathrm{m}}$ for some nonnegative integer $n$ and cardinal $\mathrm{m}$.

Proof. Sufficiency follows from Theorem 2.1. Conversely, suppose ${ }^{*} \operatorname{Pext}(X, G)=0$ for all groups $X$ in 5 . By Proposition 3.2, we have $G=$ $G_{0} \oplus H$ where $G_{0} \cong \mathbb{R}^{n} \times \mathbb{T}^{\mathfrak{m}}$ for some nonnegative integer $n$ and cardinal m. Due to Lemma 3.1(ii), ${ }^{*} \operatorname{Pext}(X, H)=0$ for all groups $X$ in 5 . Then by Lemma 3.1(i), Proposition 3.4 and Proposition 3.6, $H$ is a densely divisible topological torsion group. By Theorem 2.8, $H$ can be identified with a local direct product of its $p$-components

$$
H_{p}=\left\{x \in H: \lim _{n \rightarrow \infty} p^{n} x=0\right\}
$$

belonging to different primes $p$. Assume $H \neq 0$. Then there exists a prime $p$ such that $H_{p} \neq 0$. Since the projection map $H \rightarrow H_{p}$ is continuous, $H_{p}$ is densely divisible, so by Proposition 2.9 it contains a closed subgroup $D$ such that $D \cong F_{p}$ or $D \cong \mathbb{Z}\left(p^{\infty}\right)$. In either case, $D$ is a divisible $\sigma$-compact group in $\mathcal{Q}([12,(10.5)])$. For the inclusion map $\alpha: D \rightarrow H$ and a connected group $X$ in $\mathbb{R}$, consider the exact sequence

$$
0=\operatorname{Hom}(X, H / D) \rightarrow \operatorname{Ext}(X, D) \stackrel{\alpha_{*}}{\longrightarrow} \operatorname{Ext}(X, H) .
$$

To show that $\operatorname{Ext}(X, D)=0$, let $E: 0 \rightarrow D \stackrel{\phi}{\longrightarrow} F \rightarrow X \rightarrow 0 \in \operatorname{Ext}(X, D)$. The group $F / \phi(D) \cong X$ is $\sigma$-compact since it is connected ([12, (9.14)]) and $\phi(D)$ is $\sigma$-compact, hence $F$ is $\sigma$-compact ([17, Theorem $6.10(\mathrm{c})])$ and it follows that every group $F[n]$ is $\sigma$-compact. By Lemma 3.3, $\alpha_{*}(E)=\alpha E$ 
is a $*$-pure extension, so it splits. Since $\alpha_{*}$ is injective, $E$ splits as well and we obtain $\operatorname{Ext}(X, D)=0$. But then Theorem 2.6 shows that $D$ is connected, a contradiction. Consequently, $H=0$ and we have $G \cong$ $\mathbb{R}^{n} \times T^{m}$, as desired.

\section{Injective and projective properties}

A group $G$ in $\mathfrak{R}$ is called pure injective in $\mathbb{R}$ if it has the injective property relative to all pure extensions in $\mathbb{R}$, that is, if for every pure proper exact sequence $0 \rightarrow A \rightarrow B \rightarrow C \rightarrow 0$ in $\mathfrak{\Omega}$ and every $\alpha \in \operatorname{Hom}(A, G)$ there is a $\beta \in \operatorname{Hom}(B, G)$ such that the diagram

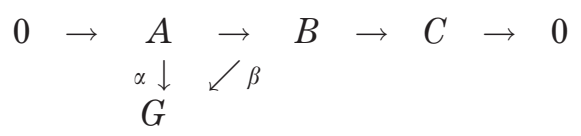

is commutative. Similarly, we call a group in $\mathbb{R} *$-pure injective in $\mathfrak{R}$ if it has the injective property relative to all $*$-pure extensions. Then we have:

THEOREM 4.1. The following are equivalent for a group $G$ in $\mathbb{R}$ :

(1) $G$ is *-pure injective in $\mathbb{2}$;

(2) ${ }^{*} \operatorname{Pext}(X, G)=0$ for all groups $X$ in $\mathbb{2}$;

(3) $G \cong \mathbb{R}^{n} \oplus \mathbb{T}^{\mathfrak{m}}$ where $n$ is a nonnegative integer and $\mathfrak{m}$ is a cardinal.

Proof. Suppose that $G$ is $*$-pure injective in 2 . Then every $*$-pure extension $0 \rightarrow G \rightarrow B \rightarrow X \rightarrow 0$ splits because there is a commutative diagram

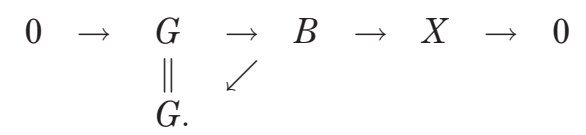

Consequently, (1) implies (2). By Theorem 3.7, (2) implies (3). The groups of the form $\mathbb{R}^{n} \times{ }^{\mathrm{m}}$ are injective in $\mathfrak{R}$ (Theorem 2.1), hence (3) implies (1).

By the theorem above, the $*$-pure injectives in $\mathbb{I}$ are exactly the injectives in $\mathfrak{R}$. As an immediate consequence, we obtain a complete description of the pure injectives in 2 . This extends [14, Theorem 2.7] and shows that the result on discrete and compact injectives in $\mathbb{2}$ as stated in [7, Proposition 8] is incorrect. 
COROLLARY 4.2. The following are equivalent for a group $G$ in $\mathfrak{R}$ :

(1) $G$ is pure injective in $\mathfrak{\Omega}$;

(2) $\operatorname{Pext}(X, G)=0$ for all groups $X$ in $\mathfrak{2}$;

(3) $G \cong \mathbb{R}^{n} \oplus T^{\mathrm{m}}$ where $n$ is a nonnegative integer and $\mathrm{m}$ is a cardinal.

Recall that a proper exact sequence $E: 0 \rightarrow A \rightarrow B \rightarrow C \rightarrow 0$ in $\mathfrak{R}$ is said to be topologically pure if the induced sequence

$$
0 \rightarrow \overline{n A} \rightarrow \overline{n B} \rightarrow \overline{n C} \rightarrow 0
$$

is proper exact for all positive integers $n$ (see [13]). Pontrjagin duality shows that the sequence $E$ is topologically pure if and only if its dual sequence

$$
0 \rightarrow \widehat{C} \rightarrow \widehat{B} \rightarrow \widehat{A} \rightarrow 0
$$

is *-pure (see [13, Corollary 2.6]). We call a group $G$ in $\mathfrak{R}$ topologically pure projective in $\Omega$ if it has the projective property relative to all topologically pure extensions, in other words, if for every topologically pure exact sequence $0 \rightarrow A \rightarrow B \rightarrow C \rightarrow 0$ and every $\gamma \in \operatorname{Hom}(G, C)$ there is a $\delta \in \operatorname{Hom}(G, B)$ such that the diagram

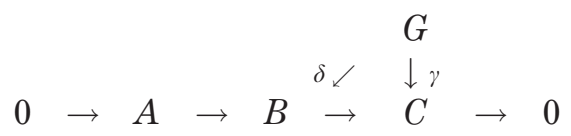

is commutative. Then dualization of Theorem 4.1 yields the following result which extends [13, Theorem 4.4(3)]:

COROLlary 4.3. The following are equivalent for a group $G$ in $\mathfrak{\Omega}:$

(1) $G$ is topologically pure projective in $\mathbf{2}$;

(2) every topologically pure sequence $0 \rightarrow A \rightarrow B \rightarrow G \rightarrow 0$ splits;

(3) $G \cong \mathbb{R}^{n} \oplus \bigoplus_{\mathfrak{m}}$ Z where $n$ is a nonnegative integer and $\mathfrak{m}$ is a cardinal.

Acknowledgments. The author would like to thank the referee for the valuable comments and suggestions which helped to improve this paper.

\section{REFERENCES}

[1] D. L. Armacost, The Structure of Locally Compact Abelian Groups, Marcel Dekker Inc., New York, 1981.

[2] J. BRACONNIER, Sur les groupes topologiques localement compacts, J. Math. Pures Appl., N.S. 27 (1948), pp. 1-85. 
[3] J. DixmieR, Quelques propriétés des groupes abéliens localement compacts, Bull. Sci. Math. (2) 81 (1957), pp. 38-48.

[4] D. N. Dikranjan, I. R. Prodanov and L. N. Stoyanov, Topological Groups: Dualities and Minimal Group Topologies, Marcel Dekker Inc., New York, Basel, 1989.

[5] R. D. Engelking, General Topology, revised and completed edition, Heldermann Verlag, Berlin, 1989.

[6] L. Fuchs, Infinite Abelian Groups, Vol. I, Academic Press, New York, 1970.

[7] R. O. FulP, Homological study of purity in locally compact groups, Proc. London Math. Soc. 21 (1970), pp. 501-512.

[8] R. O. FulP, Splitting locally compact abelian groups, Michigan Math. J. 19 (1972), pp. 47-55.

[9] R. O. FULP and P. GRIFFITH, Extensions of locally compact abelian groups I, Trans. Amer. Math. Soc. 154 (1971), pp. 341-356.

[10] R. O. FulP and P. GRIFFITH, Extensions of locally compact abelian groups II, Trans. Amer. Math. Soc. 154 (1971), pp. 357-363.

[11] P. A. GRIffith, Infinite Abelian Group Theory, The University of Chicago Press, Chicago and London (1970).

[12] E. HewitT and K. Ross, Abstract Harmonic Analysis, Vol. I, second edition, Springer Verlag, Berlin, 1979.

[13] P. Loth, Topologically pure extensions, in: Abelian Groups, Rings and Modules, Proceedings of the AGRAM 2000 Conference in Perth, Western Australia, July 9-15, 2000, Contemp. Math. 273, Amer. Math. Soc. (2001), pp. 191-201.

[14] P. Loth, Pure extensions of locally compact abelian groups, Rend. Sem. Mat. Univ. Padova 116 (2006), pp. 31-40.

[15] M. Moskowitz, Homological algebra in locally compact abelian groups, Trans. Amer. Math. Soc. 127 (1967), pp. 361-404.

[16] L. C. Robertson, Connectivity, divisibility and torsion, Trans. Amer. Math. Soc. 128 (1967), pp. 482-505.

[17] M. Stroppel, Locally Compact Groups, EMS Textbooks in Mathematics, Eur. Math. Soc., Zürich, 2006.

[18] N. Vilenkin, Direct decompositions of topological groups I, Mat. Sb., N. S. 19 (61) (1946), pp. 85-154. [English translation from the Russian by E. Hewitt in A.M.S. Translations, Series 1, Volume 23, Providence, Rhode Island (1950).]

Manoscritto pervenuto in redazione il 9 agosto 2013. 